\title{
3D GRAY-LEVEL HISTOMORPHOMETRY OF TRABECULAR BONE - A METHODOLOGICAL REVIEW
}

\author{
ZBISŁAW TABOR ${ }^{\bowtie, 1}$ AND ZBIGNIEW LATAŁA ${ }^{2}$ \\ ${ }^{1}$ Institute of Teleinformatics, Cracow University of Technology, ul. Warszawska 24, 31-155 Kraków, Poland; \\ ${ }^{2}$ Institute of Applied Informatics, Cracow University of Technology, Al. Jana Pawła II 37, 31-864 Kraków, \\ Poland \\ e-mail: ztabor@pk.edu.pl; zlatala@mech.pk.edu.pl \\ (Received January 2, 2014; revised January 31, 2014; accepted February 7, 2014)
}

\begin{abstract}
The goal of standard histomorphometry is to provide methods of qualitative description of tissue structure based on image data. Typical measurements include geometric areas, perimeters, length, angle of orientation, form factors, center of gravity coordinates etc. There are well-established procedures for deriving the aforementioned quantities from binary images. However, segmentation of in vivo images of trabecular bone poses a problem which has not been solved yet. Recent years have brought significant developments within an emerging field of "gray-level histomorphometry". The general goal of gray-level histomorphometry is to provide procedures for measuring geometric areas, perimeters, length, angle of orientation, form factors, center of gravity coordinates etc. without the need for image segmentation. Although the field is not very mature yet, the collected results suggest that this approach opens new perspectives which should not be overlooked by the scientific community. In the present review we summarize the state-of-the-art within the 3D gray-level histomorphometry.
\end{abstract}

Key words: gray-level images, histomorphometry, trabecular bone

\section{INTRODUCTION}

World Health Organization (WHO) defines osteoporosis as "a systemic skeletal disease characterized by low bone mass, deterioration of trabecular architecture and increased fragility of bone" (WHO, 1994). Accordingly, the primary determinant of the risk of osteoporotic fracture is bone density assessed using densitometric methods like bone mineral density (BMD) or bone mineral content (BMC). Osteoporosisrelated decrease of bone mass influences especially trabecular bone, the bony tissue constituting interior of vertebral bodies or epiphyses of long bones.

Although decreased value of BMD or BMC is, according to $\mathrm{WHO}$, the primary symptom of osteoporosis, $55 \%$ to $70 \%$ osteoporotic fractures are observed in patients with normal BMD, which thus do not conform to the diagnostic criterion recommended by WHO (Wainwright et al., 2005). It also follows from the laboratory experiments that only from $40 \%$ to $80 \%$ of the variance of the mechanical parameters values like Young's modulus or ultimate stress can be explained by the variance of the values of bone density. It is argued that the factor responsible for the unexplained part of the variance of the values of me- chanical parameters is the spatial organization of bony tissue (microarchitecture) (Ciarelli et al., 1991; Latała et al., 2013).

As reported by the Surgeon General of the USA (http://www.surgeongeneral.gov), about 10 million Americans over age 50 have osteoporosis, while another 34 million are at risk. Each year about 1.5 million people suffer from an osteoporotic-related fracture. Hip fractures are associated with increased risk of mortality, which is $2.8-4$ times greater among hip fracture patients during the first 3 months after the fracture, than among the individuals who do not suffer from a fracture. Due primarily to aging of the population, the number of hip fractures in the United States could double or even triple by the year 2040 .

Because osteoporosis influences especially the trabecular bone, there is a challenge to develop methods of trabecular bone characterization, which would be complementary to BMD, applicable in clinical diagnosis and thus based on 2D (DXA or radiographic projections) or preferentially on 3D (either CT or MRI) images of trabecular bone.

Recent years have brought significant developments within an emerging field of "gray-level histomorpho- 
metry". The goal of standard histomorphometry is to provide methods of qualitative description of tissue structure based on image data. Typical measurements include geometric areas, perimeters, length, angle of orientation, form factors, center of gravity coordinates etc. There are well-established procedures for deriving the aforementioned quantities from binary images. However, segmentation of in vivo images of trabecular bone poses a problem which has not been solved yet. This is the point where the gray-level histomorphometry comes into play.

The general goal of gray-level histomorphometry is to provide procedures for measuring geometric areas, perimeters, length, angle of orientation, form factors, center of gravity coordinates etc. without the need for image segmentation. Although the field is not very mature yet, the collected results suggest that this approach opens new perspectives which should not be overlooked by the scientific community. In the present review we summarize the state-of-the-art within the $3 \mathrm{D}$ gray-level histomorphometry. Open problems and future perspectives of the field are also sketched.

\section{METHODS OF ACQUISITION OF 3D TRABECULAR BONE IMAGES}

High resolution 3D images of trabecular bone can be acquired with micro computed tomography (microCT). Commercially available microCT scanners offer images with resolution ranging from 1 micrometer at the cost of a large X-rays dose and long time of data acquisition. Although microCT scanners are appropriate only for in vitro experiments, the microCT image data provide gold standards for comparison with images acquired with clinical devices.

Three-dimensional images of trabecular bone can also be captured with clinical CT scanners (Fig. 1). CT scans are currently used to assess mineral and volumetric density of the trabecular and cortical bone compartments (Lang et al., 1999) what requires calibration of the image data with respect to a special phantom. The resolution of such images is however markedly worse than that of microCT. Actually, the best resolution achievable for axial skeleton is about 200 micrometers in the plane of the scan, which is approximately the same as the thickness of trabeculae. The resolution in the direction perpendicular to the scan plane is even worse, equal to about $0.5 \mathrm{~mm}$. Better resolution up to 80 microns at tolerable radiation doses can be obtained with peripheral CT (Muller, 2003) but CT scanners of this type are designed to examine bones only at peripheral skeletal sites: the radius and the tibia. Safe dose of X-rays and the duration time of the image acquisition process are the factors limiting in-vivo CT resolution.

Modern strong field clinical MRI scanners provide spatial resolution of trabecular bone images of up to 100 microns in plane and slice thickness as low as 250 microns. Using small scanners with high field strengths even higher spatial resolution can be obtained up to isotropic voxel sizes of 50 microns (Fig. 2). A serious disadvantage of high resolution MRI is a long acquisition time of up to $10-20 \mathrm{~min}$. Fast gradients and dedicated coils with a small field of view are also required as well as a high signal-to-noise ratio. Due to those limitations as well as the motion artifacts in the axial skeleton, high resolution MRI is applicable only to peripheral sites.

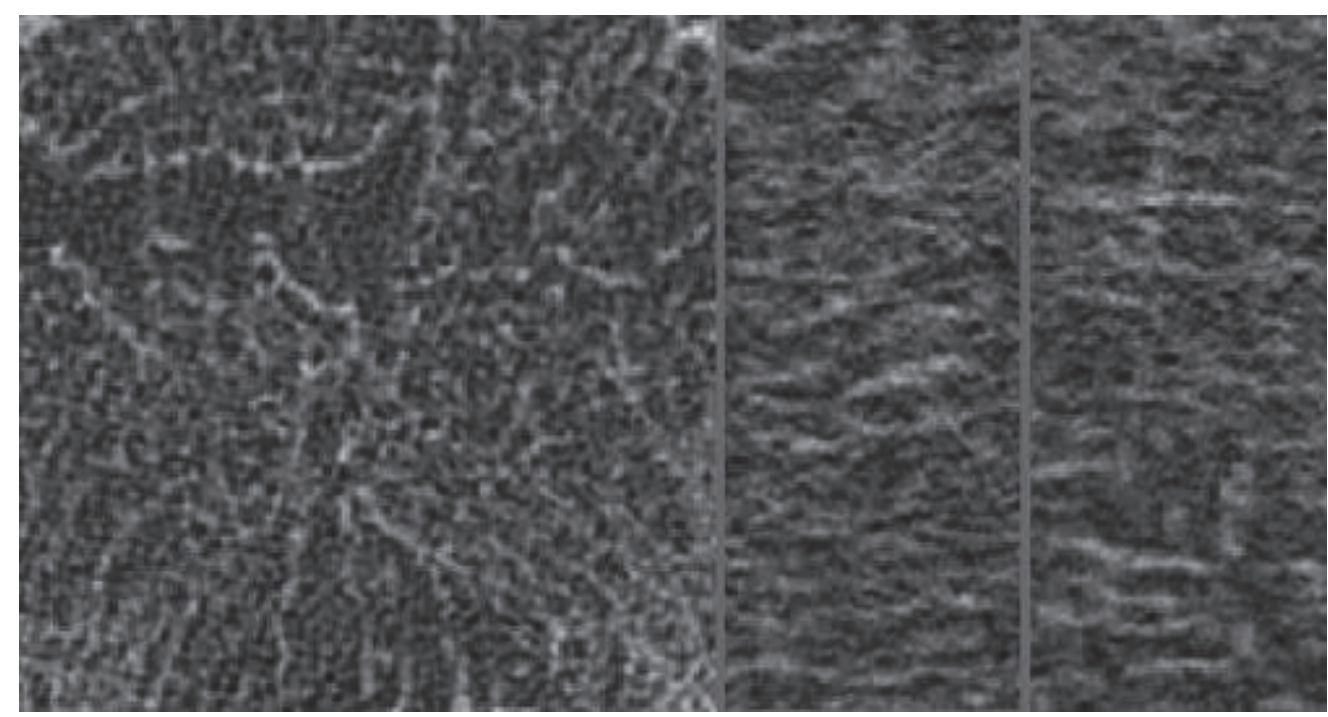

Fig. 1. Three perpendicular sections of a 3D CT image of a vertebral trabecular bone sample. 


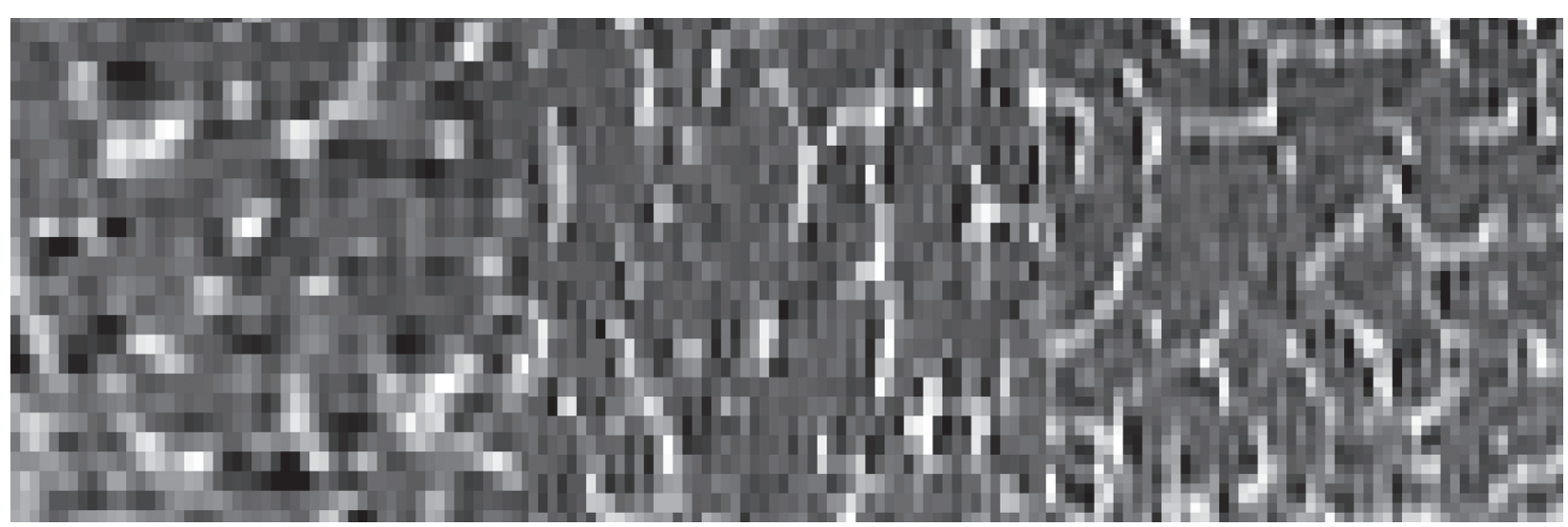

Fig. 2. Three perpendicular sections of a negative of a high resolution MRI image of a distal radius trabe-cular bone sample. Due to low proton content trabe-culae are seen as bright bars.

Summarizing, 3D images of trabecular bone samples are now easily available. The examinations of peripheral sites deliver images with resolution comparable to the typical thickness of trabeculae. The resolution of the 3D images of axial skeleton and femoral neck is however markedly worse. Because the correlation between trabecular bone features at different skeletal sites is often mild, the methods must be worked out to quantify trabecular bone structure from low-resolution data. In the following chapter parameters used to characterize trabecular bone based on high-resolution images are first presented.

\section{HISTOMORPHOMETRY OF TRABECULAR BONE}

Before standard histomorphometry measurement procedures can be applied, image data must be segmented yielding binary images with, by convention, white representing bone and black representing marrow space. The segmentation of a grey-level image of a trabecular bone sample is a separate problem. For highresolution data segmentation is usually performed on the base of a grey-level histogram by setting a threshold at a minimum between two maxima corresponding to bone and marrow phases. By contrast, there is no generally accepted segmentation algorithm, used to process low-resolution images of trabecular bone.

The trabecular bone volume fraction (BV/TV) is a surrogate measure of the bone density and equals to the ratio of bone voxels to the number of all voxels within the image.

Trabecular thickness (Tb.Th) is an average over local measurements of bony tissue thickness taken at randomly selected bone voxels. The local thickness at a given point of a trabecular bone is the diameter of the maximal sphere that contains above mentioned point and is included entirely within the tissue of interest (Hildebrand and Rüegsegger, 1997). Trabecular separation ( $\mathrm{Tb} . \mathrm{Sp})$ is the thickness of the marrow phase. The trabecular number Tb.N can also be calculated with the above approach. For this purpose trabecular bone image is skeletonized and diameters of the maximal spheres fitting the background of the skeleton are found for randomly selected points. Then, Tb.N is taken as the inverse of the average of the diameters of the spheres.

Estimates of $\mathrm{Tb} . \mathrm{Th}, \mathrm{Tb} . \mathrm{Sp}$ and $\mathrm{Tb} . \mathrm{N}$ are rarely calculated from the definition. As an alternative, a distance transform is applied to the phase of interest and then the values of the transform are sampled along the skeleton of the phase and used as estimates of the local thickness.

Given the skeleton of the trabecular structure, nodestrut analysis (Garrahan et al., 1986) of the skeleton can be conducted. From the image of the skeleton the node number (N.Nd), termini number (N.Tm), nodeto-node strut count Nd-Nd CS, node-to-terminus strut count Nd-Tm CS, terminus-to-terminus strut count $\mathrm{Tm}-\mathrm{Tm} \mathrm{SC}$ and different ratios like the node-terminus ratio $(\mathrm{Nd} / \mathrm{Tm})$ are calculated to quantify the trabecular structure. Clearly, the method is sensitive to the features of the skeletonization algorithm and artifacts of skeletonization can bias the estimated values of the computed parameters, among which N.Tm can be especially severely influenced.

Trabecular bone pattern factor (TB.Pf) (Hahn et al., 1992) is another method of quantifying the structure of trabecular bone. For 3D datasets, the area of the bone-marrow interface (BS) and the volume of the bony tissue (BV) are measured. Then dilation is applied to the image resulting in thickening the trabecular structure by 1 voxel. The bone area BS' and the bone volume BV' are calculated for the dilated image. The trabecular bone pattern factor is defined as: 


$$
\mathrm{TB} \cdot \mathrm{Pf}=\frac{\mathrm{BS}^{\mathrm{B}}-\mathrm{BS}^{\prime}}{\mathrm{BV}-\mathrm{BV}^{\prime}} .
$$

Well connected networks result in low or even negative TB.Pf. Poorly connected networks result in high values of TB.Pf.

Structure model index (SMI) (Hildebrand and Ruegsegger, 1997) indicates the relative prevalence of rods and plates in trabecular bone structures. An ideal plate, cylinder and sphere have SMI values of 0 . 3 and 4, respectively. For trabecular structure consisting of rods and plates of the same thickness SMI is in the range from 0 and 3. The calculation of SMI is based, like calculation of TB.Pf, on dilation of a 3D image. However, in contrast to TB.Pf, the measurement of SMI is based on differential analysis of the triangulated bone surface. SMI is derived as follows:

$$
\mathrm{SMI}=6 \cdot\left(\frac{\mathrm{dBS} / \mathrm{dr} \cdot \mathrm{BV}}{\mathrm{BS}^{2}}\right) \text {. }
$$

The Euler number is an indicator of a 3D structure connectedness. The Euler number is a topologically invariant property of a three-dimensional structure. It is a measure of how many connections in a structure can be removed before the structure falls into two separate pieces. The components of the 3D Euler number are the three Betti's numbers: $\beta_{0}$ is the number of objects, $\beta_{1}$ is the connectivity, and $\beta_{2}$ is the number of cavities entirely enclosed within the examined phase. The formula for the Euler number of a $3 \mathrm{D}$ object is:

$$
\chi=\beta_{0}-\beta_{1}+\beta_{2} .
$$

Euler analysis provides a measure of connectivity density (E.Conn.D) which is equal to $\beta_{1}$ divided by the analyzed volume (Odgaard and Gundersen, 1993).

One of the most striking properties of trabecular bone is its structural anisotropy. Trabeculae are not aligned in random directions. They are rather aligned in a parallel way with the lines of major compressive or tensile stresses. Formally, structural anisotropy (fabric) is a second rank, positive definite tensor. There are a few approaches to define a fabric tensor. The most straightforward approach requires defining a vector field, which specifies locally the structure orientation.

Let $\mathbf{V}$ be a unit vector describing the mean orientation within a structure and $\mathbf{g}(\mathrm{x}, \mathrm{y}, \mathrm{z})$ be a vector describing local orientation. The error vector $\mathbf{e}(\mathrm{x}, \mathrm{y}, \mathrm{z})$ of $\mathbf{g}$ with respect to $\mathbf{V}$ is equal to $\mathbf{e}(\mathrm{x}, \mathrm{y}, \mathrm{z})=\mathbf{g}$ $\left(\mathbf{g}^{\mathrm{T}} \cdot \mathbf{V}\right) \mathbf{V}$. The total error $\mathrm{E}$ is equal to the integral of $\mathrm{e}$ over the image volume. Minimizing $\mathrm{E}$ with respect to $V$ yields an eigenequation $\mu \mathbf{V}=\lambda \mathbf{V}$ for $\mathrm{V}$, where $\mu$ is the covariance matrix of $\mathbf{g}$ (fabric tensor), i.e.:

$$
\mu_{i, j}=\int_{\Omega} g_{i} g_{j} d \omega,
$$

and $\lambda$ are the eigenvalues of the fabric tensor. The product $g_{i} g_{j}$ in Eq. 4 is integrated over the total volume of the image.

One of the typical choices of the local orientation vector field is performed on the base of the volume orientation (VO) method (Odgaard et al., 1990) (Fig. 3 ). The method works for binary images of binary structures. A local volume orientation is defined at any point within a trabecula as the orientation of the longest intercept through that point. For every point within the analyzed structure a single unit vector describing the local orientation is thus obtained. Then the components of this vector are inserted into Eq. 4 defining the VO fabric tensor.

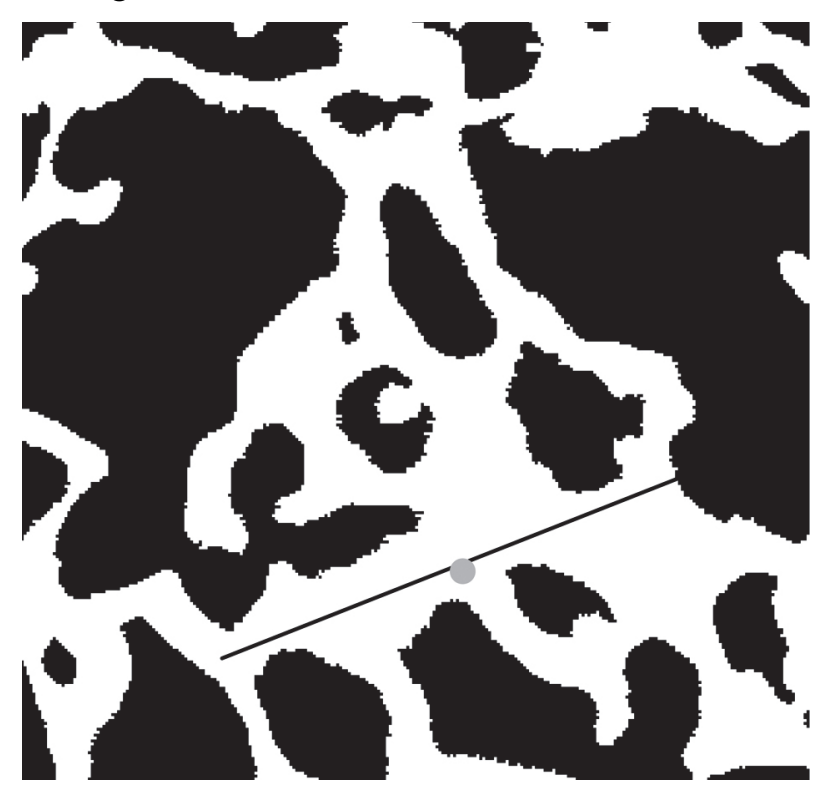

Fig. 3. Measurement of the volume orientation in a sample point (grey disk).

Another classical approach to characterize structural anisotropy is based on the mean intercept length (MIL) method (Whitehouse, 1974). The principle of the MIL method is to count the number of intersections between a family of equidistant parallel lines and the bone/marrow interface as the function of the 3D orientation $\theta$ of the family of lines (see Fig. 4 for a 2D example). The mean intercept length is the total length $\mathrm{L}_{\text {TOT }}$ of lines divided by the number of intersections $\mathrm{N}_{\mathrm{I}}(\theta)$ :

$$
\operatorname{MIL}(\theta)=\frac{\mathrm{L}_{\text {TOT }}}{\mathrm{N}_{\mathrm{I}}(\theta)} .
$$


It is often assumed that the MIL data can be approximated with an ellipsoid. This ellipsoid may be expressed by the quadratic form of a second rank tensor - the MIL fabric tensor. Histomorphometric parameters can be calculated with both commercial software offered by the manufacturers of microCT scanners or with open source applications like BoneJ plugin (Doube et al., 2010) for ImageJ (http://rsb.info.nih.gov/ij/).

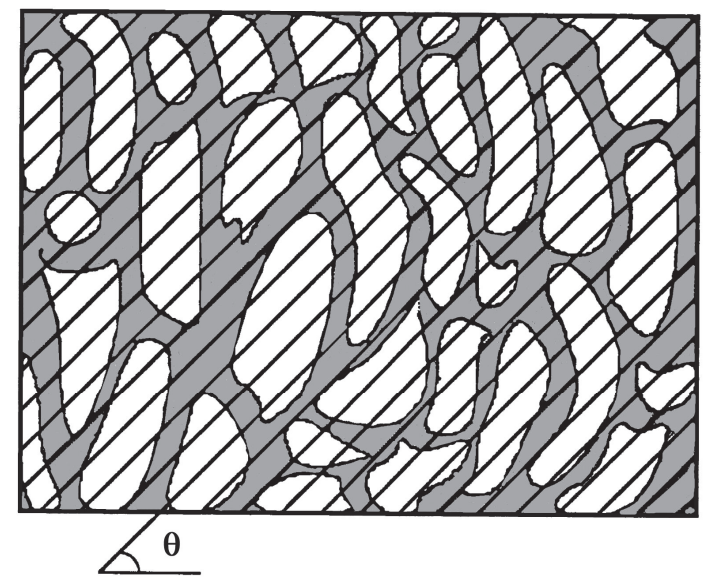

(a)
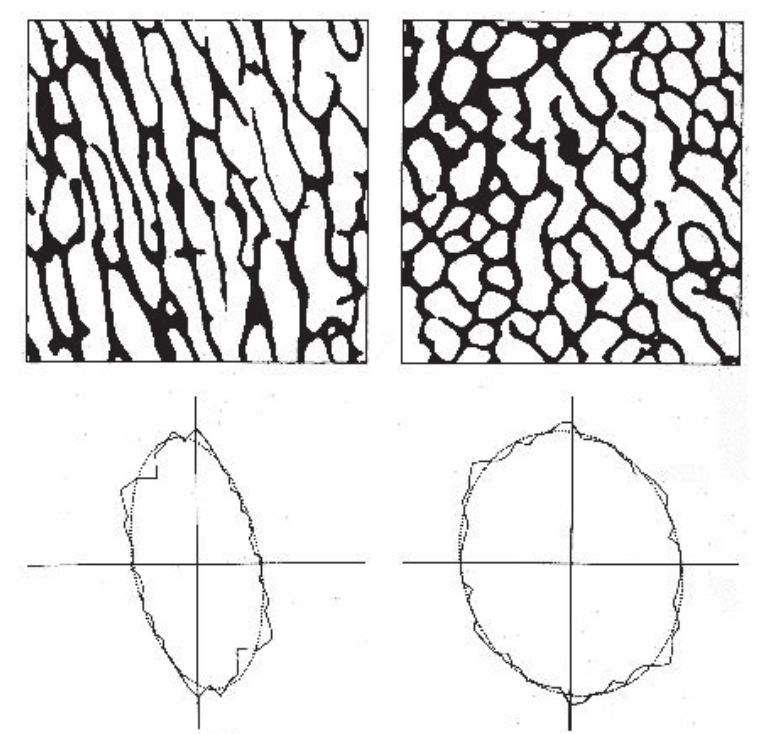

(b)

Fig. 4. The principles of the MIL measurement: (a) a linear grid imposed onto the structure, (b) MIL is a function of orientation $\theta$.

\section{GRAY-LEVEL METHODS FOR CHARACTERIZING TRABECULAR BONE STRUCTURE}

Before describing the concepts related to the graylevel histomorphometry, other gray-level based methods of characterizing trabecular structure should be men- tioned. In particular, the gray-level images of trabecular bone have been analyzed, using the methods developed for texture characterization. One of the possible approaches is based on the co-occurrence of matrix and its derived parameters (Haralick et al., 1973; Veenland et al., 1997). Firstly, histogram equalization is performed: the number of gray levels is reduced to some number $\mathrm{N}$ and gray-level intensities are redistributed in such way that the probability of occurrence for all gray-levels is equalized. Secondly, a matrix $M(d, \theta)$ is composed, so that its element $M_{i, j}(d, \theta)$ is equal to the number of the co-occurrences of gray levels $\mathrm{i}$ and $\mathrm{j}$ over a distance $\mathrm{d}$, under an angle $\theta$. To simplify calculations and avoid intensive interpolations the angle $\theta$ is typically chosen equal to integer multiples of $\pi / 4$. Normalizing the matrix elements for the total number of co-occurrences yields a probability matrix $P_{d, \theta}$. From this matrix a number of parameters can be computed, for example homogeneity HOM, contrast CON, entropy ENT, correlation COR:

$$
\begin{aligned}
& \operatorname{HOM}_{d, \theta}=\sum_{i, j=1}^{N} P_{i, j, d, \theta}^{2}, \\
& \operatorname{CON}_{d, \theta}=\sum_{i, j=1}^{N} P_{i, j, d, \theta}(i-j)^{2},(7) \\
& E^{N} T_{d, \theta}=-\sum_{i, j=1}^{N} P_{i, j, d, \theta} \ln \left(P_{i, j, d, \theta}\right), \\
& \operatorname{COR}_{d, \theta}=\frac{\sum_{i, j=0}^{N}\left(i-\mu_{i}\right)\left(j-\mu_{j}\right) P_{i, j, d, \theta}}{\sigma_{i} \sigma_{j}},
\end{aligned}
$$

where $\mu_{x}=\sum_{x=0}^{N} x, \sigma_{x}^{2}=\sum_{x=0}^{N}\left(x-\mu_{x}\right)^{2}$ with $x=i, j$.

Another approach to characterize textures of the images of trabecular bone is based on the run length method (Galloway, 1975; Cortet et al., 1999). The run method consists of counting the gray level runs in the image and expressing the statistical distribution of these runs. A gray level run is defined as a set of linearly adjacent pixels with the same gray level value. A gray level run is characterized by its gray level value $i$, its length $j$ and its direction $\theta$. For each direction matrix $\mathbf{L}_{\theta}$ is computed so that the element $\mathrm{L}_{\theta, \mathrm{i}, \mathrm{j}}$ of the matrix is equal to the number of the runs with a gray level $i$ and a length $j$. Like for co-occurrence matrix, a number of parameters can be computed from $\mathbf{L}_{\theta}$, among them short runs emphasis $R_{\theta, 1}$, long runs emphasis $R_{\theta, 2}$, gray level nonuniformity $R_{\theta, 3}$ or run length nununiformity $R_{\theta, 4}$ : 


$$
\begin{aligned}
& R_{\theta, 1}=\frac{\sum_{i=0}^{N} \sum_{j=1}^{s} \frac{L_{i, j}}{j^{2}}}{\sum_{i=0}^{N} \sum_{j=1}^{S} L_{i, j}}, \\
& R_{\theta, 2}=\frac{\sum_{i=0}^{N} \sum_{j=1}^{S} j^{2} L_{i, j}}{\sum_{i=0}^{N} \sum_{j=1}^{S} L_{i, j}}, \\
& R_{\theta, 3}=\frac{\sum_{i=0}^{N}\left(\sum_{j=1}^{S} L_{i, j}\right)^{2}}{\sum_{i=0}^{N} \sum_{j=1}^{S} L_{i, j}}, \\
& R_{\theta, 4}=\frac{\sum_{j=1}^{s}\left(\sum_{i=0}^{N} L_{i, j}\right)^{2}}{\sum_{i=0}^{N} \sum_{j=1}^{S} L_{i, j}},
\end{aligned}
$$

where $\mathrm{N}$ is the maximal gray level and $\mathrm{S}$ is the size of an image.

According to the method of differential measurement of local variations (Cortet et al., 1999), the local variations of gray levels are first estimated by the use of a differential filter (e.g., Sobel's filter) that permits an approximation of the gradient vector $\mathbf{G}(\mathrm{x}, \mathrm{y})$. Four component images are computed by convolving the original image with the Sobel's filter masks $\mathrm{G}_{0}, \mathrm{G}_{1 / 4 \pi}$, $\mathrm{G}_{1 / 2 \pi}$ and $\mathrm{G}_{3 / 4 \pi}$ in four directions $(0,1 / 4 \pi, 1 / 2 \pi, 3 / 4 \pi)$. A few parameters have been measured with this method, among them mean modulus in the horizontal direction $\mathrm{V}_{1}$, mean modulus in the vertical direction $\mathrm{V}_{2}$, differential modulus of variations $\mathrm{V}_{3}$ or scattering modulus of variations $\mathrm{V}_{4}$ :

$$
\begin{gathered}
V_{1}=\sum_{i, j=0}^{s}\left(\operatorname{Im} * G_{0}\right)_{i, j}, \\
V_{2}=\sum_{i, j=0}^{S}\left(I m * G_{1 / 2 \pi}\right)_{i, j}, \\
V_{3}=\sum_{i, j=0}^{s} G_{i, j}^{(\text {mod })}, \\
V_{4}=\frac{1}{S^{2}} \sum_{i, j=0}^{s}\left|G_{i, j}^{(\text {mod })}-V_{3}\right|,
\end{gathered}
$$

where
$G_{i, j}^{(m o d)}=\sqrt{\left(I m * G_{0}\right)_{i, j}{ }^{2}+\left(I m * G_{1 / 4 \pi}\right)_{i, j}{ }^{2}+\left(I m * G_{1 / 2 \pi}\right)_{i, j}{ }^{2}+\left(I m * G_{3 / 4 \pi}\right)_{i, j}}$

and asterisk denotes convolution and $\mathrm{S}$ is the image size.

Yet another approach to the gray level analysis of trabecular bone images is based on computing fractal dimension of trabecular structure (Pelag et al., 1983; Cortet et al., 1999). Starting from an original image $\mathrm{Im}$, an eroded $\mathrm{E}_{\varepsilon}(\mathrm{Im})$ and dilated $\mathrm{D}_{\varepsilon}(\mathrm{Im})$ versions of the image Im are obtained, using square structuring element $\varepsilon \times \varepsilon$ in size. Then the surface area parameter $\mathrm{A}(\varepsilon)$ is defined as a function of $\varepsilon$ :

$$
A(\varepsilon)=\frac{V(\varepsilon)-V(\varepsilon-1)}{2 \varepsilon},
$$

where $V(\varepsilon)=\sum_{i, j=0}^{s}\left\{D_{\varepsilon}(\operatorname{Im})_{i, j}-E_{\varepsilon}(\operatorname{Im})_{i, j}\right\}$. If $A(\varepsilon)$ can be assumed to be a power function of $\varepsilon$, that is $A(\varepsilon) \sim \varepsilon^{v}$, then the fractal dimension FD is equal to 2-v.

One may ask, how the above mentioned parameters are related to the well-known histomorphometric measures. Although the question is interesting, the answer may be difficult. In most cases multiple regression models are tested to check whether inclusion of texture parameters increases determination coefficient of parameters directly related to fracture risk e.g., fracture strength or apparent Young's modulus as compared to bone density only. The published results are not however encouraging - the texture analysis improves the prediction of mechanical parameters by at most a few percent (Veenland et al., 1997; Cortet et al., 1999).

\section{GRAY-LEVEL HISTOMORPHOMETRY}

The first attempts to derive structural information from gray-level data were based on the concept of an autocorrelation function of either an original graylevel image or - in the case of MR images - its bone volume fraction (BVF) transform (Wu et al., 1994). A $3 \mathrm{D}$ autocorrelation function $\Phi(\Delta \mathbf{r})$ is defined in a usual way:

$$
\begin{aligned}
& \Phi\left(\Delta r_{x}, \Delta r_{y}, \Delta r_{z}\right)= \\
& \quad<\operatorname{Im}(x, y, z) \cdot \operatorname{Im}\left(x+\Delta r_{x}, y+\Delta r_{y}, z+\Delta r_{z}\right)>_{x, y, z},
\end{aligned}
$$

where $\operatorname{Im}(\mathrm{x}, \mathrm{y}, \mathrm{z})$ denotes either an original gray-level intensity or BVF value at a point $(\mathrm{x}, \mathrm{y}, \mathrm{z})$ within an image and the brackets $\langle\cdot\rangle_{\mathrm{x}, \mathrm{y}, \mathrm{z}}$ indicate spatial averaging over all possible locations $(\mathrm{x}, \mathrm{y}, \mathrm{z})$.

$\Phi(\Delta \mathbf{r})$ always possesses a global maximum at $\Delta \mathbf{r}$ $=0$. For every profile of $\Phi(\Delta \mathbf{r})$ passing through the 
origin there are further maxima for a quasi-regular structure occurring at $\Delta \mathrm{r}$ equal to integer multiples of the structure's spatial period $d$. The trabecular thickness estimate Th along some profile of $\Phi(\Delta r)$ is defined (Hwang et al., 1997) as twice the value of the shift $\Delta \mathrm{r}$ so that $\Phi(\Delta r)=1 / 2 \Phi(0)$. The trabecular spacing $\mathrm{Sp}$ along the profile is defined as $\mathrm{Sp}=\mathrm{d}-\mathrm{Th}$. The structural anisotropy of trabecular bone sample can be assessed by measuring Th and $\mathrm{Sp}$ as a function of orientation. $\Phi(\Delta r)$ can be determined for all the directions and subsequently sampled at the desired angular resolution by test lines through the origin of $\Phi(\Delta r)$. Then an ellipsoid is fitted to the rose plot of $\operatorname{Th}(\theta, \varphi)$ (or $\operatorname{Sp}(\theta, \varphi))$. This ellipsoid may be expressed by the quadratic form of a second rank tensor $\mathrm{E}_{\mathrm{Th}}$ (or $\mathrm{E}_{\mathrm{Sp}}$ ). Let $\mathbf{a}_{\mathrm{th}}, \mathbf{b}_{\mathrm{th}}$ and $\mathbf{c}_{\mathrm{th}}$ denote the three eigenvectors of $E_{T h}$ so that $\left|\mathbf{a}_{\mathrm{th}}\right| \leq\left|\mathbf{b}_{\mathrm{th}}\right| \leq\left|\mathbf{c}_{\mathrm{th}}\right|$. The degree of anisotropy $\mathrm{A}_{\mathrm{Th}}$ is defined as:

$$
A_{T h}=\sqrt{1-\left(\frac{\left|a_{T h}\right|}{\left|c_{T h}\right|}\right)^{2}} .
$$

Similar expression can be written for Sp.

For structures, which are approximately isotropic within planes perpendicular to some axis (say z-axis) other parameters can be derived from autocorrelation function (Hwang et al., 1997). Having $\Phi(\Delta \mathrm{r})$ determined one may calculate the following quantities:

$$
\begin{gathered}
\mathrm{ACF}_{\text {transverse }}(\mathrm{n})=<\operatorname{Im}(x, y, z) \cdot \operatorname{Im}(x+n \cos \theta, y+n \sin \theta, z)>_{x, y, \theta, z}, \\
\operatorname{ACF}_{\text {longitudinal }}(n)=<\operatorname{Im}(x, y, z) \cdot \operatorname{Im}(x, y, z+n)>_{x, y, z},
\end{gathered}
$$

Then the transverse contiguity Tcon is defined as the ratio of the probability, so that a point from a voxel and a point from one of the voxel's eight nearestneighbors are both in bone divided by the probability that two points from the same voxel are both in bone. This ratio is approximately equal to:

$$
\text { Tcon }=\frac{A C F_{\text {transverse }}(1)}{A C F_{\text {transverse }}(0)} \text {. }
$$

Tubularity is equal to the probability that two points from adjacent voxels in the longitudinal direction are both in a bone, divided by the probability that two points from the same voxel are both in a bone. The definition of tubularity Tub is:

$$
\mathrm{Tub}=\frac{<\operatorname{lm}\left(x, y, z_{1}\right) \operatorname{Im}\left(x, y, z_{2}\right)>_{x, y, z_{1}}}{A C F_{\text {longitudinal }}(0)}
$$

where $z_{1}$ and $z_{2}$ refer to the locations of adjacent transverse sections. It has been shown in a study including 23 distal radius specimens (Hwang et al., 1997) that the autocorrelation-based parameters account for $91 \%$ of the variation in Young's modulus of the samples. Although the autocorrelation-based approach is certainly en elegant one, it can be argued that it can lead to false conclusions about structural properties in cases of structures which are not strictly periodic (Tabor, 2009).

A fuzzy-set based approach was also proposed to quantify trabecular thickness (Saha and Wehrli, 2004) and structural anisotropy (Saha and Wehrli, 2004). This approach is based on the following definitions. A digital object $O=\left(Z^{3} ; \mu_{O}\right)$ is a fuzzy subset of $Z^{3}$, where $Z$ is the set of all integers, $Z^{3}$ represents a $3 D$ digital grid and $\mu_{O}: Z^{3} \rightarrow[0 ; 1]$ specifies the membership value at each voxel. The membership value $\mu_{\mathrm{O}}$ is equal to the signal intensity within a voxel. In a binary image the distance between two points is the length of the straight-line segment joining them. In a fuzzy subset, membership values need to be accounted for in order to compute the length of a path or the distance between two points. The length of a path $\pi, \Pi_{0}(\pi)$ in a fuzzy subset $O$ is defined as the integral over fuzzy membership values along $\pi$ :

$$
\Pi_{0}(\pi)=\int_{0}^{1} \mu_{0}(\pi(t))\left|\frac{d \pi(t)}{d t}\right| d t .
$$

The fuzzy distance between two points is the mini-mum length of all paths between the two points. Sup-port of a fuzzy subset is the set of points having a nonzero fuzzy membership. If a fuzzy object has a finite support then fuzzy distance transform (FDT) can be applied to the object. FDT is an operator applied to a fuzzy subset that assigns a value at each pixel location that is the smallest fuzzy distance between the location and a point on the boundary of the support of the fuzzy subset. Trabecular thickness can be estimated by sampling FDT values along the skeleton of the fuzzy support of the structure of interest (Saha and Wehrli, 2004). Fuzzy subset-based estimates of trabecular separation can be obtained analogously (Krebs et al., 2009).

The definition of the length of a path in a fuzzy subset was also used to define a tensor of structural anisotropy. Structural anisotropy is measured in a number of candidate voxels. The fuzzy membership function is examined along sample lines, uniformly distributed over the angular space around a candidate 
voxel $\mathrm{p}$, to find an edge voxel. Specifically, the object edge point $\varepsilon_{i}^{(p)}$ on a sample line $\pi_{i}^{(p)}$ emanating from $\mathrm{p}$, is computed which Euclidean distance from p, denoted by ||$\varepsilon_{i}^{(p)}-p||$, is equal to the integration of the membership values along $\pi_{i}^{(p)}$ until a non-object point (membership value $=0$ ) is found:

$$
|| \varepsilon_{i}^{(p)}-p||=\int_{0}^{T\left(p, \pi_{i}^{(p)}\right)} \mu_{0}\left(\pi_{i}^{(p)}(t)\right)\left|\frac{d \pi_{i}^{(p)}(t)}{d t}\right| d t,
$$

where $T\left(p, \pi_{i}^{(p)}\right)$ is the parametric location of the first (from $p$ ) zero membership point on $\pi_{i}^{(p)}$. The location of the edge points is eventually corrected to ensure that the edge points found along mutually opposite sample lines are equidistant from the candidate voxel p. Finally, an ellipsoid is fitted to the rose plot of the distances ||$\varepsilon_{i}^{(p)}-p||$ averaged over a number of candidate voxels and the tensor of structural anisotropy is then defined in a usual way. The idea of sampling signal along lines distributed uniformly over the angular space around skeleton voxels in the search for boundary voxels was also used to estimate trabecular thickness and separation (Liu et al., 2013).

It is clear from the above discussion that the estimation of both fuzzy trabecular thickness and structural anisotropy requires initial preprocessing of an analyzed image to create the fuzzy subset support. For that purpose the voxels of an analyzed image, which certainly are not bone voxels, must be detected first and then assigned zero membership function value. It is not obvious that such preprocessing step is always possible for every class of the images of trabecular bone. Moreover, it has been shown that under controlled conditions, regarding resolution and noise, the FDT-based approaches do not outperform brute-force methods based on direct thickness measurement on binarized images (Petryniak and Tabor, 2012).

Other approaches to the thickness measurement have also been proposed, e.g., based on the concept of granulometry (Tabor and Petryniak, 2012; Moreno et al., 2012a). The problem is however still far from being solved. It should be noted that similar problems are addressed also within other fields of biomedical engineering, and the problems related to accurate thickness estimation are well recognized (Dougherty and Newman, 1999; Prevrhal et al., 2002; Sato et al., 2003).

Two histomorphometric parameters, which globally characterize the shape of trabecular elements are trabecular bone pattern factor (Tb.Pf) and Structure Model Index. Definitions of both of them can be reformulated to be applicable directly to gray-level images. The following notation is used (Tabor, 2011) in the derivation of the gray-level estimates of SMI. Im is an image of some structure (e.g., trabecular bone) and $\operatorname{Im}(\mathrm{x})$ denotes gray-level intensity at a point $\mathrm{x} . \operatorname{Im}$ eroded by a structuring element $\varepsilon$ is denoted $\varepsilon \mathrm{Im}$. V and $\mathrm{S}$ are the structure volume and the structure surface area, respectively. $\varepsilon \mathrm{V}$ and $\varepsilon \mathrm{S}$ are the volume and the surface area of the eroded structure. Consider a discrete, binary image of some structure (black background, white structure). Let the structure be eroded by an eroding element $\varepsilon$ approximating a ball with a unit radius (e.g., a $3 \times 3$ pixels square in $2 \mathrm{D}$ ). Then $\mathrm{V}-\varepsilon \mathrm{V}$, which is the number of white pixels in the difference image Im- $\mathrm{Im}$, approximates the structure surface area $S$. Similarly, $S-\varepsilon S$ approxi-mates up to a multiplicative constant $\mathrm{dSV} / \mathrm{dr}$. Hence one has:

$$
\mathrm{SMI}=6 \cdot \frac{d \mathrm{~S}_{\mathrm{V}}}{\mathrm{dr}} \cdot \frac{\mathrm{V}_{\mathrm{v}}}{\mathrm{S}_{\mathrm{V}}^{2}} \approx 6 \cdot \frac{1-\varepsilon \mathrm{S} / \mathrm{S}}{1-\varepsilon \mathrm{V} / \mathrm{V}} .
$$

Because we also have $V=\int_{\Omega} \operatorname{Im}(\mathrm{x}) \mathrm{dV}$ and $S=\int_{\Omega}|\nabla \operatorname{Im}(x)| d V$, we finally get:

$$
\mathrm{SMI}=6 \cdot \frac{1-\frac{\int_{\Omega}|\nabla \varepsilon \operatorname{lm}(\mathrm{x})| \mathrm{dV}}{\int_{\Omega}|\nabla \operatorname{lm}(\mathrm{x})| \mathrm{dV}}}{1-\frac{\int_{\Omega} \varepsilon \operatorname{lm}(\mathrm{x}) \mathrm{dV}}{\int_{\Omega} \operatorname{Im}(\mathrm{x}) \mathrm{dV}}} .
$$

The operators on the right hand side for Eq. 28 involve summations over signal intensities and gradient magnitudes in image voxels and thus - in contrast to triangulation-based definition of (Hildebrand and Ruegsegger, 1997) - can be directly applied to gray-level images. It has been shown (Tabor, 2011) that SMI definition, given in Eq. 28 is equivalent to standard approach if applied to binary data (correlation between a standard and an alternative approaches equal to 0.97). Eq. 28 has not been however tested on low-resolution medical data so far, although it has been shown that this approach is robust with respect to noise and resolution decrease (Tabor, 2011). Other triangulation-free definitions of SMI were also proposed (Ohser et al., 2009) but their form renders their direct application to gray-level data.

The above sketched approach can be also applied to derive a definition of Tb.Pf, applicable to graylevel images. Note that formally, translating the structure surface by a small extent $d r$ in its normal direction, 
Tb.Pf can be defined as the ratio of the derivative of structure surface $\mathrm{S}$ with respect to $\mathrm{dr}$ and $\mathrm{S}$, that is $\mathrm{Tb} . \mathrm{Pf}=\mathrm{d}(\ln (\mathrm{S})) / \mathrm{dr}$, where an approxi-mation for $\mathrm{S}$ has been given above.

The MIL measurement can be generalized to graylevel images (Tabor and Rokita, 2007; Tabor, 2009; 2012; Moreno et al., 2012b). The MIL measurement involves counting the number of intersections between a family of parallel, equidistant lines and the bone-marrow interface. The inter-line distance $d$ is usually considered to be a user-adjusted parameter. The MIL measurement can be generalized to gray-level images if a limit $d \rightarrow 0$ is taken. Indeed, assume that the interface between two phases contains only lines with slope $\theta$ and the total length L (Fig. 5). Then the number $\mathrm{N}(\alpha)$ of intersections between a family of lines characterized by the slope $\alpha$ and the boundary is equal to:

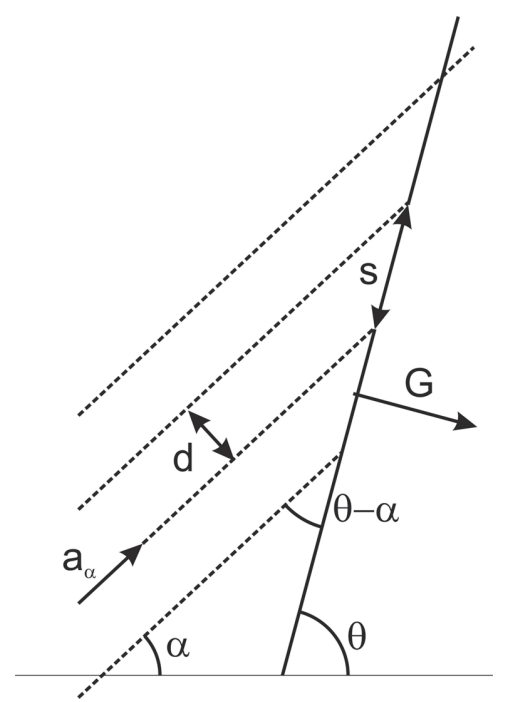

Fig. 5. The MIL measurement for a boundary composed entirely of a single line with slope $\theta$. The distance $s$ between the successive intersections is equal to $d / \sin (\theta-\alpha)$.

$$
N(\alpha)=\frac{L}{d} \sin (\theta-\alpha) .
$$

Because for the binary images the magnitude of the gradient $\mathbf{G}$ is also constant along the phase interfaces, it can be normalized without loss of generality to the unit length. Hence one has:

$$
N(\alpha)=\frac{L}{d}\left|G \cdot a_{\alpha}\right|,
$$

where $\mathrm{a}_{\alpha}$ is a unit vector with orientation equal to $\alpha$. The absolute value of the scalar product $G \cdot a_{\alpha}$ is taken because the MIL measurement is invariant with respect to the change of the gradient direction $\mathbf{G}$ by $\pi$. For a square region of size $\mathrm{S}$ the total length of lines characterized by the slope $\alpha$ and shifted by $\mathrm{d}$ is equal to $\mathrm{S}^{2} / \mathrm{d}$. Thus for a boundary model as above, $\operatorname{MIL}(\alpha)$ is equal to:

$$
\operatorname{MIL}(\alpha)=\frac{S^{2}}{L\left|G \cdot a_{\alpha}\right|} .
$$

For the case of an interface composed of lines with arbitrary slopes we have:

$$
\operatorname{MIL}(\alpha)=\frac{\int_{\Omega} d \omega}{\int_{\Omega}\left|G \cdot a_{\alpha}\right| d \omega}=\frac{1}{\left\langle\left|G \cdot a_{\alpha}\right|\right\rangle_{\Omega}},
$$

where $\langle\cdot\rangle_{\Omega}$ denotes averaging of an argument over the region of interest $\Omega$. The definition of MIL given in Eq. 32 can be directly generalized to gray-level images. An efficient method to calculate MIL based on the above presented gray-level approach has been published in Moreno et al., 2012b.

Besides MIL, there are other methods to quantify structural anisotropy from gray-level images but it appears that a method based on calculating the graylevel structure tensor has been studied most frequently. Interestingly, it has been shown that in 2D MIL and GST tensors are related through an analytical formula (Tabor, 2009):

$$
\mathrm{MIL}=\mathrm{K}_{1} \cdot \mathrm{GST}+\mathrm{K}_{2} \cdot \operatorname{det}\left(\mu_{\mathrm{GST}}\right) \cdot \mathrm{GST}^{-1},
$$

where $K_{1}$ and $K_{2}$ are some constants. Consequently, the principal orientation and principal values of one of the tensors can be computed while the principal orientation and principal values of the other tensor are known. The estimation of GST is however computationally much less expensive than the computation of MIL. Unfortunately, there are some drawbacks of computing GST if the resolution of an analyzed image is not isotropic (Tabor, 2010) or the quality of the data is too low (Tabor et al., 2013). For example (Tabor, 2010), GST can demonstrate false anisotropy and wrong orientation if the voxel size is not isotropic, as is the case of in vivo CT data. In spite of these drawbacks it has been shown (Tabor et al., 2013) that GST performs significantly better than MIL when applied to clinical data.

Other approaches to quantify structural anisotropy include deriving an equivalent to an inertia tensor, where the signal intensity plays the role of mass (Varga and Zysset, 2009; Vasilić et al., 2009) or the Hessian matrix (i.e., the matrix of second derivatives) of the signal intensity (Eberly et al., 1994) but the usefulness of these methods for the assessment of structural anisotropy is not still well recognized. Interestingly, the principal values of inertia tensor can 
be used to locally estimate thickness of structural elements and classify their shape into either rods or plates (Vasilić et al., 2009). The method, which is a direct development of the concept of digital topological analysis (Wehrli et al., 2001) works well for gray-level data with quality substantially better than clinically achievable.

\section{COMPARISON OF STANDARD AND GRAY LEVEL HISTOMORPHOMETRY METHODS}

Comparison of standard and gray level imagebased measurements has been demonstrated in a few articles only. Autocorrelation function-derived surrogate measures of trabecular thickness and separation, introduced by Hwang et al. (1997) were not compared against standard measures. Fuzzy distance transform (FDT) - based estimate of Tb.Th, introduced in the work of Saha and Wehrli (2004b) was compared with standard methods in the work of Petryniak and Tabor (2012). This study was based on microCT images of 25 samples of distal radius trabecular bone, acquired with a resolution equal to $34 \mu \mathrm{m}$. The mean (standard deviation) values of standard $\mathrm{Tb}$.Th and FDT-based $\mathrm{Tb}$.Th were equal to $110 \pm 10 \mu \mathrm{m}$ and $79 \pm 10 \mu \mathrm{m}$. The values of Pearsons coefficient of correlation between standard $\mathrm{Tb}$.Th and FDT-based Tb.Th was equal to 0.99 . The slope of standard Tb.Th vs. FDTbased $\mathrm{Tb}$.Th plot was equal to $1.03 \pm 0.04$, what suggested that the difference between the two measures corresponded to some shift only. These authors also tested how the FDT-based estimate of Tb.Th, calculated for low-resolution, noisy images is related to gold standard measurement, based on microCT data. They reported quite high correlation coefficient (about 0.8) even for 5-fold decreased reso-lution and an intensive noise. A comparison between microCT and clinical CT data was not however demon-strated so far.

FDT-based estimates of trabecular separation were compared vs. standard estimates in the work of Krebs et al. (2009). It was shown that both methods are strongly correlated (correlation coefficient equal to 0.99), when applied to high resolution data (microCT images, 82 micron pixel size) and the results of both measurements span the same range. A substantial overestimation of FDT-based estimate of Tb.Sp was however found, compared to gold standard microCT, when applying FDT-based method to HRCT images (resolution equal to $156 \times 156 \times 400 \mu \mathrm{m}$ ) of trabecular bone. In this case the coefficient of correlation dropped down to 0.94 , standard measurements spanned range from 399 to $140 \mu \mathrm{m}$, while FDT-based measurements spanned range from 795 to $1596 \mu \mathrm{m}$.
Another method of calculating Tb.Th and Tb.Sp from gray-level images was proposed in the work of Liu et al. (2013). The method, which uses a star-line tracing technique is claimed to effectively deal with partial volume effects of in vivo imaging where voxel size is comparable to trabecular thickness. Although the authors presented some numerical results of their computations, no comparison with standard methods of estimating either $\mathrm{Tb} . \mathrm{Th}$ or $\mathrm{Tb} . \mathrm{Sp}$ was actually presented.

In the work of Tabor (2011) standard and graylevel based methods of computing structure model index SMI were compared, based on a set of 25 microCT images of distal radius trabecular bone, acquired with resolution equal to $34 \mu \mathrm{m}$. The mean (standard deviation) values of standard SMI and a gray level-based SMI were equal to $2.38 \pm 0.38$ and $2.31 \pm 0.42$. The values of Pearsons coefficient of correlation between standard SMI and gray levelbased SMI was equal to 0.97. Based on BlandAltman analysis, the authors demonstrated that both methods are equivalent within $95 \%$ limits of agreement. It was also tested how the gray level-based estimate of SMI, calculated for down-scaled, noisy images is related to gold standard measurement, based on microCT data. It was found that the SMI estimate (either standard or gray-level based) grows with growing voxel size but quite high correlation coefficient (about 0.8 ) even for 5-fold decreased resolution and intensive noise was observed. A comparison between estimates of SMI from microCT and clinical CT data was not however done.

Standard and gray level-based methods of estimating structural anisotropy were compared in a few articles (Tabor, 2009; 2012; Tabor et al., 2013). In the case of two-dimensional data the equivalence of MIL and GST was proven analytically, as already mentioned (Tabor, 2009). The equivalence of both methods in 3D was tested based on a set of 25 microCT images of distal radius trabecular bone, acquired with resolution equal to $34 \mu \mathrm{m}$ (Tabor, 2012). It was shown that the coefficient of correlation between corresponding eigenvalues of standard and gray level-based fabric tensor was very high, and exceeded 0.98. The principal anisotropy directions were calculated for the MIL and the GST fabric tensors. It was reported that the coefficient of spherical correlation between the first anisotropy direction of the MIL fabric tensor and the third anisotropy direction of the GST tensor was equal to 0.78 , the coefficient of spherical correlation between the second anisotropy directions of the MIL and the GST tensors was equal to 0.97 and the coefficient of 
spherical correlation between the third anisotropy direction of the MIL fabric tensor and the first anisotropy direction of the GST tensor was equal to 0.94 . The Pearson's coefficient of correlation between degree of anisotropy (equal to the ratio of the largest and the smallest eigenvalues of the fabric tensor) of GST and degree of anisotropy of MIL was equal to 0.90 .

Comparison between structural anisotropy parameters calculated for microCT and for CT images of trabecular interior of whole vertebral bodies was reported in the work of Tabor et al. (2013). CT images were acquired with resolution $137 \mu \mathrm{m} \times 137 \mu \mathrm{m}$, slice thickness $0.75 \mathrm{~mm}$ and reconstruction interval equal to $0.1 \mathrm{~mm}$. It was found that the correlation between eigenvalues of MIL and GST calculated for microCT data is not as good as in the case of small samples and did not exceeded 0.89. Correlation between GST parameters estimated for high and low-resolution data was mild (absolute value of the correlation coefficient not larger than 0.62). Even worse low results were found for the MIL method (absolute value of the correlation coefficient not larger than 0.50). A comparison between structural MIL anisotropy parameters estimated from microCT and GST parameters calculated for clinical CT data was not done yet.

\section{CONCLUSION}

Problems related to gray-level histomorphometry attach increasing attention of scientific community. The current research is focused primarily on developing robust gray-level methods of quantifying structural anisotropy and it appears that the gold standard MIL approach will be replaced by a novel standard in a near future (Moreno et al., 2012a). The methods of estimating other histomorphometric parameters from gray-level data are much less developed. Gray-level data-based methods of estimation of trabecular thickness and separation, presented so far, require substantial pre-processing, which involves either image segmentation or determining bone-marrow interfaces - steps difficult to accomplish in the case of in-vivo data. Gray-level data-based methods of estimation of structure model index or trabecular bone pattern factor, although existing, have not been tested on data characterized by quality comparable to achievable invivo. Gray-level data-based methods of estimating other parameters, e.g., Euler number density have not even been proposed. The solution to the above listed problems is extremely important because the incorporation of accurate structural information is crucial for the success of emerging patient-specific finite element analyses of fracture risk (Trabelsi and Yosibash, 2011).

\section{ACKNOWLEDGEMENTS}

This work was supported by Polish Ministry of Science and High Education as the research project No. N N518 423536.

\section{REFERENCES}

Ciarelli MJ, Goldstein SA, Kuhn JL, Cody DD, Brown MB (1991). Evaluation of orthogonal mechanical properties and density of human trabecular bone from the major metaphyseal regions with materials testing and computed tomography. J Orthop Res 6:674-82.

Cortet B, Dubois P, Boutry N, Bourel P, Cotten A, Marchandise X (1999). Image analysis of the distal radius trabecular network using computed tomography. Osteoporosis Int. 9:410-9.

Doube M, Kłosowski MM, Arganda-Carreras I, Cordeliéres F, Dougherty RP, Jackson J, et al. (2010). BoneJ:free and extensible bone image analysis in ImageJ. Bone 47:1076-9.

Dougherty G, Newman D (1999). Measurement of thickness and density of thin structures by computed tomography:A simulation study. Med Phys 26:1341-8.

Eberly D, Gardner R, Morse B, Pizer S, Scharlach C (1994). Ridges for image analysis. J Math Imaging Vis 4:35373.

Galloway MM (1975). Texture analysis using grey level run lengths. Comput Graph Image Process. 4:172-9.

Garrahan NJ, Mellish RWE, Compston JE (1986). A new method for the two-dimensional analysis of bone structure in human iliac crest biopsies. J. Microsc. 142: 341-9.

Hahn M, Vogel M, Pompesius-Kempa M, Delling G (1992). Trabecular bone pattern factor - a new parameter for simple quantification of bone microarchitecture. Bone $13: 327-30$.

Haralick RM, Shanmugam K, Dinstein I (1973). Textural features for image classification. IEEE Trans Syst Man Cybern 3:510-612.

Hildebrand T, Ruegsegger P (1997). Quantification of bone microarchitecture with the structure model index. Comp Meth Biomech Biomed Eng 1:15-23.

Hildebrand T, Rüegsegger PA (1997). New method for the model-independent assessment of thickness in threedimensional images. J Microsc 185:67-75.

Hwang SN, Wehrli FW, Williams JL (1997). Probabilitybased structural parameters from three-dimensional nuclear magnetic resonance images as predictors of trabecular bone strength. Med Phys 24:1255-61.

Krebs A, Graeff C, Frieling I, Kurz B, Timm W, Engelke K, Glüer CC (2009). High resolution computed tomography of the vertebrae yields accurate information on trabecular distances if processed by 3D fuzzy segmentation approaches. Bone 44:145-52.

Lang TF, Li J, Harris ST, Genant HK (1999). Assessment 
of vertebral bone mineral density using volumetric quantitative CT. J Comput Assist Tomogr 23:130-7.

Latała Z, Czerwiński E, Tabor Z, Petryniak R, Konopka T, (2013). Structural derminations of mechanical behavior of whole human L3 vertebrae- an ex vivo microCT study. Proceeding of Vipimage 2013-IV Eccomas Thematic Conference on Computational Vision and Medical Image Processing, Funchal, Portugal, October 14-16: 377-81.

Liu Y, Jin D, Saha PKA (2013). New algorithm for trabecular bone thickness computation at low resolution achieved under in vivo condition. 2013 IEEE 10th International Symposium on Biomedical Imaging: From Nano to Macro, San Francisco, CA, USA, April 7-11.

Moreno R, Borga M, Smedby Ö (2012a). Estimation of trabecular thickness in gray-scale images through granulometric analysis. Proc SPIE 8314, Medical Imaging 2012: Image Processing, 831451.

Moreno R, Borga M, Smedby Ö (2012b). Generalizing the mean intercept length tensor for gray-level images. Med Phys 39:4599-612.

Muller R (2003). Bone microarchitecture assessment - current and future trends. Osteoporosis Int 14 (suppl. 5): S89.

Odgaard A, Gundersen HJG (1993). Quantification of connectivity in cancellous bone, with special emphasis on 3-D reconstructions. Bone 14:173-82.

Odgaard A, Jensen EB, Gundersen HJG (1990). Estimation of structural anisotropy based on volume orientation. A new concept. J Microsc 157:149-62.

Ohser J, Redenbach C, Schladitz K (2009). Mesh free estimation of the structure model index. Image Anal Stereol 28:179-85.

Pelag S, Naor J, Hartley R, Anvir D (1983). Multiple resolution texture analysis and classification. IEEE Trans Patt Anal Machine Intell 6:661-74.

Petryniak R, Tabor Z (2012). Surrogate measures of thickness in the regime of limited image resolution. Part 1: Fuzzy distance transform. Lecture notes in computer science 7268:318-26.

Prevrhal S, Shepherd JA., Genant HK (2002). Accuracy of CT-based thickness measurement of thin structures: Modeling of limited spatial resolution in all three dimensions. Med Phys 30:1-8.

Saha PK, Wehrli FW (2004a). A robust method for measuring trabecular bone orientation anisotropy at in vivo resolution using tensor scale. Pattern Recogn 37:1935-44.

Saha PK, Wehrli FW (2004b). Measurement of trabecular bone thickness in the limited resolution regime of in vivo MRI by fuzzy distance transform. IEEE Trans Med Imaging 23:53-62.

Sato Y, Tanaka H, Nishii T, Nakanishi K, Sugano N, Kubota T, Nakamura H, et al. (2003). Limits on the accuracy of 3-D thickness measurement in magnetic resonance images-effects of voxel anisotropy. IEEE Transactions on Medical Imaging 22:1076-88.
Tabor Z (2009). On the equivalence of two methods of determining fabric tensor. Med Eng Phys 31:1313-22.

Tabor Z (2010). Anisotropic resolution biases estimation of fabric from $3 \mathrm{~d}$ gray-level images. Med Eng Phys $32: 39-48$

Tabor Z (2011). A novel method of estimating structure model index from gray-level images. Med Eng Phys $33: 218-25$.

Tabor Z (2012). Equivalence of mean intercept length and gradient fabric tensors - 3D study. Med. Eng. Phys. 34: 598-604.

Tabor Z, Petryniak R (2012). Surrogate measures of thickness in the regime of limited image resolution. Part 2: Granulometry. Lecture notes in computer science 7268:355-61.

Tabor Z, Petryniak R, Latała Z, Konopka T (2013). The potential of multi-slice computed tomography based quantification of the structural anisotropy of vertebral trabecular bone. Med Eng Phys 35:7-15.

Tabor Z, Rokita E (2007). Quantifying anisotropy of trabecular bone from gray-level images. Bone 40:966-72.

Trabelsi N, Yosibash Z (2011). Patient-specific finite-element analyses of the proximal femur with orthotropic material properties validated by experiments. J Biomed Eng 33:061001.

Varga P, Zysset PK (2009). Sampling sphere orientation distribution:an efficient method to quantify trabecular bone fabric on grayscale images. Medical Image Analysis 13:530-41.

Vasilić B, Rajapakse CS, Wehrli FW (2009). Classification of trabeculae into three-dimensional rodlike and platelike structures via local inertial anisotropy. Med Phys 36: 3280-91.

Veenland JF, Link TM, Konermann W, Meier N, Grashuis JL, Gelsema ES (1997). Unraveling the role of strength and density in determining vertebral bone strength. Calcif Tissue Int 61:474-9.

Wainwright SA, Marshall LM, Ensrud KE, Cauley JA, Black DM, Hillier TA, et al. (2005). Hip fracture in women without osteoporosis. J Clin Endocrinol Metab 90:2787-93.

Wehrli FW, Gomberg BR, Saha PK, Song HK, Hwang SN, Snyder PJ (2001). Digital topological analysis of in vivo magnetic resonance microimages of trabecular bone reveals structural implications of osteoporosis. J Bone Miner Res 16:1520-31.

Whitehouse WJ (1974). The quantitative morphology of anisotropic trabecular bone. J Microsc 101:153-68.

World Health Organization (1994). Assessment of fracture risk and its application to screening for postmenopausal osteoporosis. Technical Report Series 843, Geneva.

Wu Z, Chung H, Wehrli FWA (1994). Bayesian approach to subvoxel tissue classification in NMR microscopic images of trabecular bone. Magn Reson Med 31:3028. http://www.surgeongeneral.gov 